\title{
Improving Validity of Cause of Death on Death Certificates
}

\author{
Ryan A. Hoffman \\ Department of Biomedical Engineering \\ Georgia Institute of Technology \\ Atlanta, GA, USA \\ rhoffman12@gatech.edu
}

\author{
Janani Venugopalan \\ Department of Biomedical Engineering \\ Georgia Institute of Technology \\ Atlanta, GA, USA \\ jvenugopalan3@gatech.edu
}

\author{
Li Qu \\ Georgia Inst. of Technology, Emory \\ University, and Peking University \\ Atlanta, GA, USA \\ quliyutu@gatech.edu
}

\author{
Hang $\mathrm{Wu}$ \\ Department of Biomedical Engineering \\ Georgia Institute of Technology \\ Atlanta, GA, USA \\ hangwu@gatech.edu
}

\author{
Prof. May D. Wang \\ Department of Biomedical Engineering \\ Georgia Institute of Technology \\ Atlanta, GA, USA \\ maywang@gatech.edu
}

\begin{abstract}
Accurate reporting of causes of death on death certificates is essential to formulate appropriate disease control, prevention and emergency response by national health-protection institutions such as Center for disease prevention and control (CDC). In this study, we utilize knowledge from publicly available expert-formulated rules for the cause of death to determine the extent of discordance in the death certificates in national mortality data with the expert knowledge base. We also report the most commonly occurring invalid causal pairs which physicians put in the death certificates. We use sequence rule mining to find patterns that are most frequent on death certificates and compare them with the rules from the expert knowledge based. Based on our results, $20.1 \%$ of the common patterns derived from entries into death certificates were discordant. The most probable causes of these discordance or invalid rules are missing steps and non-specific ICD-10 codes on the death certificates.
\end{abstract}

\section{CCS CONCEPTS}

- Applied computing Health informatics • Computing methodologies Rule learning

\section{KEYWORDS}

Public Health Informatics, Sequential Pattern Mining, Mortality Reporting

\footnotetext{
" Permission to make digital or hard copies of all or part of this work for personal or classroom use is granted without fee provided that copies are not made or distributed for profit or commercial advantage and that copies bear this notice and the full citation on the first page. Copyrights for components of this work owned by others than ACM must be honored. Abstracting with credit is permitted. To copy otherwise, or republish, to post on servers or to redistribute to lists, requires prior specific permission and/or a fee. Request permissions from Permissions@acm.org.
}

$A C M-B C B^{\prime} 18$, August 29-September 1, 2018, Washington, DC, USA

(c) 2018 Association for Computing Machinery.

ACM ISBN 978-1-4503-5794-4/18/08...\$15.00

https://doi.org/10.1145/3233547.3233581
ACM Reference format:

Ryan A. Hoffman, Janani Venugopalan, Li Qu, Hang Wu, and May D. Wang. 2018. Improving Validity of Cause of Death on Death Certificates. In Proceedings of 9th ACM International Conference on Bioinformatics, Computational Biology and Health Informatics, Washington, DC, USA, August 29 - September 1, 2018 (ACM-BCB'18), 6 pages. https://doi.org/10.1145/3233547.3233581

\section{INTRODUCTION}

Approximately 2.6 million deaths occur each year in the United States (US) and 56 million deaths occur per year worldwide [1, 2]. Accurate death statistics are imperative to help the national health protection institutions such as the National Center for Health Statistics prevent epidemics and disease outbreak, formulate a response to communicable diseases and evaluate statistics such as the birth and death trends. The data from death certificates is also used for the estimation of the trends in chronic conditions such as the prevalence of diabetes and cardiovascular conditions. Causes of death from death records are often used by the reporting agencies to carry out the tasks mentioned above. To ensure a timely and accurate response to disease threats, high-quality death information on death certificates is essential.

The World Health Organization (WHO) has classified the causes of death (COD) using the International Statistical Classification of Diseases and Related Health Problems 10th Revision (ICD-10) which contains 22 chapters covering 2,046 categories of diseases [3, 4]. Despite the pressing need for high quality cause of death information, challenges such as lack of adequate knowledge and practice still exist for the accurate filling of death certificates. These challenges lead to death certificates of uncertain quality. Studies have found disagreements between the death certificate COD and the sequence of events reported in the medical record[5, 6]. The reporting of COD with a higher accuracy is difficult to implement in some situations, i.e. when the certifying physician is not the primary care provider, when the primary care provider 
may be part of a separate healthcare organization or located in a different area, when certificate completion is significantly delayed after the death, or when the certifying physician does not have ready access to relevant medical records. These situations are part of the reality of medical practice, where the clinicians providing care the time of a patient's death may not have a comprehensive knowledge of the patient's medical history. In fact, the errors in COD reports results from improperly maintained and improved data quality within both systems in state and local hospitals. One study found that only $56.9 \%$ of attending physicians, $56.0 \%$ of resident physicians, and $55.7 \%$ of medical students matched experts for the correct cause of death in clinical case studies [7]. Another study found that $45 \%$ of resident respondents incorrectly identified a cardiovascular event as the primary cause of death [8]. The Framingham Heart Study and other studies have indicated that coronary artery disease is overestimated on death certificates as a cause of death in the general population by $24 \%$ and two times more in older patients than in younger ones [9]. The extent of such overestimation affects the quality of death certificate information and national mortality statistics is not known.

The information on COD constitutes the basis of health systems in hospitals and is essential for studying the relationships among diseases. The inaccurate reporting of these data could lead to inappropriate public health interventions [10]. Moreover, discrepancies in vital statistics documents pose a challenge in implementing effective public health interventions and accurate reporting of morbidity and mortality information $[11,12]$. For example, Lauren E. Johns et. al. have shown that inaccurate COD reports on health disparity have a large impact on New York City premature cardiovascular mortality [12]. Accurate scientific results have a strong need for improving the accuracy and validity of mortality statistics $[13,14]$. In addition, accurate COD reports are also important in clinical trials and studies. Besides, COD reports are the basis for the National Center for Health Statistics (NCHS) to help with surveillance of disease and proper allocation of funds for public health programs and research, and to help prioritize governmental decisions and actions regarding health care. Because health statistics, national mortality and morbidity statistics, and data on disease prevalence in society are largely derived from COD reports, the accuracy of COD reports is essential.

Although ICD provides detailed and specific rules, COD reports still have many issues. Some studies have focused on the coding problems that are related to particular diseases in specific countries, such as hypertension [15], myocardial infarction [16]. Some studies have come up with the incompleteness and inaccuracy in cause-of-death statement [17]. Several researchers have studied patient medical charts for errors in cause-of-death reporting, and several have demonstrated inaccurate COD reporting among residents [8]. A few studies have discussed the types of coding errors and the reasons for them [18]. No study has provided effective solutions to the errors in the COD reports. Methods to remedy errors in COD reports are urgently needed. To increase the accuracy of COD reports, physician training in death certificate completion is found effective [17]. The NYC
Office of Vital Statistics is solving this issue, especially on heart disease death reporting. An intervention was implemented within eight NYC hospitals from 2009 to 2010. The proportion of heart disease deaths reported at the intervention hospitals decreased $50 \%$ to the level of those at the nonintervention hospitals after the intervention [19, 20]. Many low-cost or free methods are also used, including an e-learning module created by New York City DOHMH in cooperation with the National Association for Public Health Statistics and Information Systems, and cause-of-death documentation handbooks provided by the CDC [21, 22]. However, these methods can only reduce the possibility of errors when recording COD reports, but not avoid or find the errors in the COD reports after recording the COD reports.

There is a gap in research about the efficient determination of the true match among several potential matches for COD reports [23]. This process is extremely complex and time intensive. Luciana K.T. et.al. identified accurate deaths focusing on only anaphylaxis-caused deaths in Brazil [24]. Perviz A. M. et.al. revealed that only $10-15 \%$ of assigned underlying causes in data from England are not true pathophysiological causes of death. Although this research showed that the COD reports have some inaccurate records, they focus on only one disease in a specific country or area. Another research study focused on accurate cause classification for only stillbirths and neonatal deaths [25]. There are also studies providing new methods for reclassification of the underlying cause of death using all the COD codes including the errors [26].

In order to document acceptable causal relationships to be used in automated and manual mortality coding, experts NCHS have published a comprehensive list of acceptable sequence codes for the cause of death [27]. The list consists of valid causes of death and the list of ICD-10 codes which can cause the COD code. Accuracies on the death certificates can be improved by multiple ways, one of which is to captured concordance with this expert pool of knowledge. However, studies have not actively used this resource to identify the discordances in codes put on death certificates, their sources and help improve the current clinical practice regarding the filling of death certificates.

In this paper, we utilized this publicly available resource of expert knowledge to determine the discordance in death certificates in national mortality repositories (National Vital Statistics System (NVSS) death certificate data). To determine frequently filled patterns from NVSS data, we used sequential rule mining. Then we compared the rules obtained from the sequential rule mining with those obtained from the expert knowledge base. The goal is to develop processes for eliminating the wrong and inaccurate COD records. In addition, our methods will help researchers better understand the relationships among diseases as well as the right intervention of public health.

We structure the rest of the paper as follows: we first describe the data sources and modeling in section 2 , followed by the results and discussion in section 3 . Finally, we conclude with the conclusions, limitations, and potential for future work in section 4 . 


\section{EXPERIMENTAL AND COMPUTATIONAL DETAILS}

As mentioned above, this study seeks to find the extent of discordances in the death certificates in national mortality database with an expert pool of knowledge, the most common sources of differences, and the sources of these discordances. We used the death certificates from those made public by the NVSS.

\subsection{Data Sources: Expert Knowledge Base}

Medical experts working on the COD have published a comprehensive list of acceptable sequence codes for the cause of death, which integrates NCHS's guidance as well as other international sources of data [28]. This data consists of a valid cause of death relationships between ICD-10 codes. Each chain given consists of an address (COD) (F3) followed by one or two sub-addresses (F2: F1). The relationships are such that each address is caused by the following sub-address, i.e. F2 $\rightarrow$ F3. In case of more than one sub-address, the address is caused by the following sub-address and all the sub-addresses which fall in between the two (F1: F2 $\rightarrow$ F3).

In this dataset, some of the relationships that are marked ambivalent with ambivalent codes are defined. Relationships which are marked ambivalent indicate that further clarifications may be needed in the future. In this analysis, we utilized all the relationships, including the ones marked as ambivalent.

\subsection{Data Sources: Death Certificate Data}

National Vital Statistics System (NVSS), coordinated by the National Center for Health Statistics aggregates the causes of death for all deaths occurring within the United States from 1959 to 2014 [2]. For this analysis, we used the mortality data from 2012, which contains 2,547,864 deaths. Each death certificate format in vital statistics offices of each state, the District of Columbia, and other special jurisdictions vary but generally consists of the underlying cause of death as recorded by physicians and other details such as the demographics, comorbid conditions, race and ethnicity. The cause of death on the death certificates was recorded as entity access codes and record access codes (up to 20 conditions). The entity axis codes refer to raw data put on death certificates and the record access axis codes refer to the codes cleaned by the NVSS. The entity access codes for this data contains two parts, with part one containing the ordered set of COD (sequences) codes, and part two containing additional related COD codes, which are unordered. Since the goal of this study is to find the discrepancies in the sequence of COD on the actual death certificates (not cleaned or filtered codes), we used the part one of the entity access codes for this analysis. Using the COD information, we extracted rules indicative of the most frequently used sequences on COD using sequential rule mining.

\subsection{Deriving Frequent COD Patterns from Death Certificates}

In this analysis, we derive the most frequently used patterns from death certificates using sequence rule mining (SRM).
Sequence rule mining, sequence pattern mining [29-33] or association rule mining $[34,35]$ are the most commonly used temporal models in literature for finding temporal relationships among sequences. SRM has diverse pattern mining applications in finance and market analysis [36, 37], travel analysis [38], mobile learning [39] and database projections. (PrefixSpan [40], MEMISP [41]). In healthcare, SRM has applications in multidimensional EEG analysis [42], administrative data analysis [43], heart disease prediction [34, 35], healthcare auditing [44], and neurological diagnosis [45]. It was first introduced by Agrawal et al. to extract regularities between products in large-scale warehouse databases [46]. Ordonez et al. adopted SRM in medical data and proposed an improved algorithm to constrain rules so as to speed up the mining process [35]. In this analysis, SRM was chosen as the method for analysis as opposed to direct comparison of the sequences on the death records, since SRM discovers the sequences which are more commonly used on the death certificates. This helps the clinicians and the national health institutions find top sequences where discordances occur with the expert knowledge base described above. This allows for targeted interventions for clinician training and clinical decision support systems. SRM is used to discover all temporal sequences frequently found in the dataset. The rules are determined useful if there exists a minimum presence in the dataset. The rules are included for analysis if they have a minimum support. Support of a rule is defined as the proportion of sequences in the data that exhibit the pattern [47]. In COD data mining, a rule of the form "X => Y, with a support B" can be interpreted as follows. If the sequence has $\mathrm{COD} \mathrm{X}$, there is $\mathrm{B} \%$ possibility of it being followed by COD Y. In the current NVSS dataset, which does not have temporal relationships, the pattern mining is performed on the sequences of COD in the death certificates.

The training dataset in the death certificates consists of a list of COD $C=\left[C_{1}, C_{2}, \ldots, C_{K}\right]$. Using this training dataset, we discover a set of $N$ rules, $S=\left[R_{1}, R_{2}, R_{3} \ldots R_{N}\right]$. Each rule $R$ in the set of rule $S$ is given by $R=<r_{1}, r_{2}, \ldots, r_{T}>$, such that $r_{1}, r_{2}, \ldots r_{N}$ is the sequence of COD in the rule $R$. They are sequentially ordered to reflect the relationship of $r_{1}->r_{2}->r_{3} \ldots r_{T}$ (T is the number of COD in the sequence). The support of a rule $R$ in the set of sequences $S$ is defined as the number of sequences that contain this rule. The support value in this analysis is used as a metric to pick the valid rules, which have a value larger than a minimum support. In our experiments, we use the BIDE algorithm, short for BI-Directional-Extension-based frequent closed sequence mining, proposed by Wang et al. [48]. The BIDE algorithm was selected because it is an implementation of frequent closed sequence mining which emphasizes scalability and real-world performance.

\subsection{Deriving Rules from Expert Knowledge}

In the expert-derived relationship data we mentioned above, a total of 10,849 unique ICD-10 codes were found. In addition, we also have a list of ICD-10 codes, which were marked as not valid in the US for mortality reporting (1,301 out of 10,849). In this analysis, after consultation with experts from the Center for Disease Control and Prevention, we used only the codes which 
were valid in the US. Following that consultation, we excluded from analysis death records containing external (V-Y) or nature of injury (S-T) codes, as being outside of the scope for physician mortality certifiers.

\section{RESULTS AND DISCUSSION}

\subsection{Results from SRM Analysis}

We applied BIDE to the cause of death data to part 1 of the entity access codes from 2012 death certificates.
Table 1: Size of Frequent Patterns from SRM Analysis

\begin{tabular}{lc}
\hline Length & Count \\
\hline 2 & 8368 \\
3 & 3386 \\
4 & 61 \\
$5+$ & 0 \\
\hline
\end{tabular}

After performing the filtering procedure in section 2.4, 224,608 death records were omitted from the analysis. From the remaining records, using a minimum support of 50 occurrences,

Table 2: Top 10 Invalid Rules of Length 2

\begin{tabular}{lc}
\hline Support & Rule (c1->c2) \\
\hline 7278 & Essential (primary) hypertension $->$ Unspecified diabetes mellitus without complications \\
7175 & Chronic obstructive pulmonary disease, unspecified $->$ Malignant neoplasm of bronchus or lung, unspecified \\
6598 & Congestive heart failure $->$ Chronic obstructive pulmonary disease, unspecified \\
6248 & Congestive heart failure $->$ Atherosclerotic heart disease \\
5998 & Essential (primary) hypertension $->$ Chronic obstructive pulmonary disease, unspecified \\
5992 & Chronic obstructive pulmonary disease, unspecified $->$ Atherosclerotic heart disease \\
5670 & Atherosclerotic heart disease $->$ Chronic obstructive pulmonary disease, unspecified \\
5665 & Essential (primary) hypertension - Unspecified dementia \\
3550 & Atrial fibrillation and flutter $->$ Atherosclerotic heart disease \\
3407 & Essential (primary) hypertension $->$ Non-insulin-dependent diabetes mellitus without complications \\
\hline
\end{tabular}

Table 3: Top 10 Invalid Rules of Length 3

\begin{tabular}{lc}
\hline Support & Rule (c1->c2) \\
\hline 1329 & Essential (primary) hypertension $->$ Unspecified diabetes mellitus without complications $->$ Cardiac arrest, unspecified \\
1284 & Congestive heart failure $->$ Atherosclerotic heart disease $->$ Cardiac arrest, unspecified \\
1186 & Chronic obstructive pulmonary disease, unspecified $->$ Atherosclerotic heart disease $->$ Cardiac arrest, unspecified \\
1079 & Essential (primary) hypertension $->$ Unspecified diabetes mellitus without complications $->$ Atherosclerotic heart disease \\
879 & Essential (primary) hypertension $->$ Unspecified diabetes mellitus without complications $->$ Acute myocardial infarction, \\
unspecified \\
861 & Congestive heart failure $->$ Chronic obstructive pulmonary disease, unspecified $->$ Respiratory failure, unspecified \\
798 & Essential (primary) hypertension $->$ Chronic obstructive pulmonary disease, unspecified $->$ Cardiac arrest, unspecified \\
733 & Atherosclerotic heart disease $->$ Chronic obstructive pulmonary disease, unspecified $->$ Cardiac arrest, unspecified \\
607 & Congestive heart failure $->$ Chronic obstructive pulmonary disease, unspecified $->$ Cardiac arrest, unspecified \\
600 & Chronic obstructive pulmonary disease, unspecified $->$ Malignant neoplasm of bronchus or lung, unspecified $->$ \\
\end{tabular}

Table 4: Top 10 Valid Rules of Length 2

\begin{tabular}{lc}
\hline Support & Rule $(\mathrm{c} 1->\mathrm{c} 2)$ \\
68064 & Atherosclerotic heart disease $->$ Cardiac arrest, unspecified \\
47089 & Atherosclerotic heart disease $->$ Acute myocardial infarction, unspecified \\
38386 & Atherosclerotic heart disease $->$ Congestive heart failure \\
30814 & Congestive heart failure $->$ Cardiac arrest, unspecified \\
30787 & Essential (primary) hypertension $->$ Cardiac arrest, unspecified \\
28401 & Chronic obstructive pulmonary disease, unspecified $->$ Respiratory failure, unspecified \\
27733 & Pneumonia, unspecified $->$ Septicemia, unspecified \\
26349 & Pneumonia, unspecified $->$ Respiratory failure, unspecified \\
24728 & Essential (primary) hypertension $->$ Atherosclerotic heart disease \\
24535 & Acute myocardial infarction, unspecified $->$ Cardiac arrest, unspecified
\end{tabular}


we extracted 11,815 sequential rules, together accounting for 4,010,150 causal relationships between codes. Of these rules, 61 had length 4 and 3,386 had length 3, with the remaining 8,368 being length 2 , seen in Table 1 .

\subsection{Results from Comparing Rules from SRM with Expert Knowledge}

For each rule of length greater than two found using SRM, we checked for the validity using the relationship dataset mentioned above. Rules were mapped as invalid if the address and subaddress from SRM were not found in the relationship data set. For multi-part rules, if one part of the SRM rule did not conform, the entire rule was marked invalid, as seen in Fig. 1.

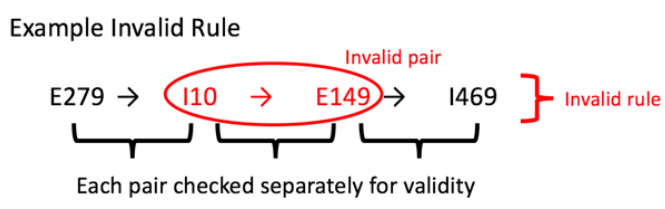

Figure 1: Interpretation of a multi-part rule, where any invalid link identifies the rule as invalid.

Of the total 11,815 rules, $2,378(20.1 \%)$ of the rules were marked as invalid. Based on the counts, there the cumulative count of relationships from SRM was 4,010,150. Of these relationships, a cumulative count of 491,955 (12.3\%) were marked invalid. Rules can be invalid if any individual step is invalid or if the ICD10 code not allowable or is not specific enough. Table 2 and Table 3 show the top rules, of lengths two and three respectively, which were marked invalid based on the frequency of occurrence. Support is expressed in the number of records which contained the rule. The full table of invalid rules is available at miblab.bme.gatech.edu. For comparison, valid rules are shown in Table 4 . The valid rules show relationships that are accepted by medical consensus as being plausibly causally linked.

In summary, $20.1 \%$ of the frequently occurring patterns from the death certificate data showed discordance with the expert knowledge data. Major causes of this could include the use of non-specific codes and missing entities in the sequences entered. Finding the codes which are top areas of discordance on the COD section of death certificates can help the national health institutions such the CDC, NCHS, and NVSS provide the certifying clinicians with the requisite training to avoid potential inaccuracies. They could also help these institutions to assist the physicians to record a more detailed information. Also, finding out the missing links in the COD sequence for death analysis has the potential to help in clinical decision support.

\section{CONCLUSIONS}

The lack of methods and evaluation of the accuracy of current death records in national mortality databases is a challenge and can result in inappropriate public health interventions, loss of life, or increased expense. In this study, we develop a framework to showcase the discordances in COD coding of death certificates in mortality databases with an expert knowledge base. We also identified the rules with the most frequent discrepancies. This provides us with the knowledge to improve the training of physicians to improve the filling of death certificates. It also gives us an insight into the common discordances and the systemic changes required for improving the accuracy of death certificates. These systematic differences may highlight potential opportunities for updating and revising either clinician training or the causal classification procedures themselves. In addition, this can be incorporated into intelligent analytics with future potential for improving the accuracy of death reporting.

There are limitations to the methods and results described by this work. Though potential root causes for the discordance are proposed and discussed throughout, the lack of ground truth hampers direct evaluation of the causes of the discordance. Additionally, only one year of historical data was used for the rule mining step. In the future, we will extend our analysis to data from spanning multiple years. We will also use graph based ontology analysis which can potentially provide the missing links to the clinicians at the time of filling of the death certificates. We believe that this work demonstrates the applicability and value of decision support systems to mortality reporting, and hope that future work in this area may definitively identify the sources of the sources of these errors. We will also investigate the combination of our methods with decision support systems.

\section{ACKNOWLEDGMENTS}

This work was carried out in collaboration with the Center for Disease Control and Prevention. This work was supported in part by grants from the National Center for Advancing Translational Sciences of the National Institutes of Health (NIH) under Award UL1TR000454 to Dr. May D. Wang, National Science Foundation Award NSF1651360, and the US Department of Health and Human Services (HHS) Centers for Disease Control and Prevention (CDC) HHSD2002015F62550B to Dr. May D. Wang, and Microsoft Research and Hewlett Packard. This article does not reflect the official policy or opinions of the CDC, NSF, or the US Department of HHS and does not constitute an endorsement of the individuals or their programs.

For this work, the authors thank Paula Braun (CDC), and Charles Sirc (CDC) for their invaluable assistance and support in shaping this project. We also thank Donna Hoyert and Robert Anderson at the National Center for Health Statistics for their invaluable feedback and support.

\section{REFERENCES}

[1] Top 10 Causes of Death - Factsheet. WHO, 2013.

[2] NCHS, United States, 2014: With special feature on adults aged 55-64 (2015).

[3] International statistical classification of diseases and related health problems. World Health Organization, 2004.

[4] Mony, P. K. and Nagaraj, C. Health information management: An introduction to disease classification and coding. National Medical Fournal of India, 20, 6 (2007), 307.

[5] Sington, J. and Cottrell, B. Analysis of the sensitivity of death certificates in 440 hospital deaths: a comparison with necropsy findings. Fournal of clinical pathology, 55, 7 (2002), 499-502. 
[6] Hoff, C. and Ratard, R. Louisiana death certificate accuracy: a concern for the public's health. F La State Med Soc, 162, 6 (2010), 350-352.

[7] Messite, J. and Stellman, S. D. Accuracy of death certificate completion: the need for formalized physician training. Jama, 275, 10 (1996), 794-796.

[8] Lakkireddy, D. R., Gowda, M. S., Murray, C. W., Basarakodu, K. R. and Vacek, J. L. Death certificate completion: how well are physicians trained and are cardiovascular causes overstated? The American journal of medicine, 117, 7 (2004), 492-498.

[9] Agarwal, R., Norton, J. M., Konty, K., Zimmerman, R., Glover, M., Lekiachvili, A., McGruder, H., Malarcher, A., Casper, M. and Mensah, G. A. Peer Reviewed: Overreporting of Deaths From Coronary Heart Disease in New York City Hospitals, 2003. Preventing chronic disease, 7, 3 (2010).

[10] Seske, L. M., Muglia, L. J., Hall, E. S., Bove, K. E. and Greenberg, J. M. Infant mortality, cause of death, and vital records reporting in Ohio, United States. Maternal and child health journal, 21, 4 (2017), 727-733.

[11] Johansson, L. A. and Westerling, R. Comparing Swedish hospital discharge records with death certificates: implications for mortality statistics. International journal of epidemiology, 29, 3 (2000), 495-502.

[12] Johns, L. E., Madsen, A. M., Maduro, G., Zimmerman, R., Konty, K. and Begier, E. A case study of the impact of inaccurate cause-of-death reporting on health disparity tracking: New York City premature cardiovascular mortality. American journal of public health, 103, 4 (2013), 733-739.

[13] Madsen, A., Thihalolipavan, S., Maduro, G., Zimmerman, R., Koppaka, R., Li, W., Foster, V. and Begier, E. Peer Reviewed: An Intervention to Improve Cause-of-Death Reporting in New York City Hospitals, 2009-2010. Preventing chronic disease, 9 (2012).

[14] Gissler, M., Kauppila, R., Merilainen, J., Toukomaa, H. and Hemminki, E. Pregnancy-associated deaths in Finland 1987-1994-definition problems and benefits of record linkage. Acta obstetricia et gynecologica Scandinavica, 76, 7 (1997), 651-657.

[15] Curb, J., Babcock, C., Pressel, S., Tung, B., Remington, R. and Hawkins, C. Nosological coding of cause of death. American journal of epidemiology, 118, 1 (1983), 122-128.

[16] Guibert, R. L., Wigle, D. T. and Williams, J. I. Decline of acute myocardial infarction death rates not due to cause of death coding. Canadian journal of public health= Revue canadienne de sante publique, 80, 6 (1989), 418-422.

[17] Myers, K. A. and Farquhar, D. R. Improving the accuracy of death certification. Canadian Medical Association fournal, 158, 10 (1998), 1317-1323.

[18] Percy, C. and Muir, C. The international comparability of cancer mortality data: results of an international death certificate study. American journal of epidemiology, 129, 5 (1989), 934-946.

[19] Madsen, A. and Begier, E. Improving quality of cause-of-death reporting in New York City. Preventing chronic disease, 10 (2013).

[20] Madsen, A., Thihalolipavan, S. and Maduro, M. A successful intervention to improve the quality of cause of death reporting in New York City hospitals. (In Press). Preventing chronic disease (2017).

[21] NAPHSIS Training Resources. National Association for Public Health Statistics and Information Systems Web site.

[22] Physician's Handbook on Death Registration and Fetal Death Reporting Hyattsville, MD: Centers for Disease Control and Prevention, 2003.

[23] Skopp, N. A., Smolenski, D. J., Schwesinger, D. A., Johnson, C. J., MetzgerAbamukong, M. J. and Reger, M. A. Evaluation of a methodology to validate National Death Index retrieval results among a cohort of U.S. service members. Ann Epidemiol, 27, 6 (Jun 2017), 397-400.

[24] Tanno, L. K., Bierrenbach, A. L., Calderon, M. A., Sheikh, A., Estelle R Simons, F. and Demoly, P. Increasing the Accuracy of Notification of Anaphylaxis Deaths in Brazil through the International Classification of Diseases (ICD)-11 Revision. Fournal of Allergy and Clinical Immunology, 139, 2 (AB226.

[25] Flenady, V., Wojcieszek, A. M., Ellwood, D., Leisher, S. H., Erwich, J. J. H., Draper, E. S., McClure, E. M., Reinebrant, H. E., Oats, J. and McCowan, L. Classification of causes and associated conditions for stillbirths and neonatal deaths. Elsevier, City, 2017.

[26] Foreman, K. J., Naghavi, M. and Ezzati, M. Improving the usefulness of US mortality data: new methods for reclassification of underlying cause of death. Population health metrics, 14, 1 (2016), 14.
[27] Lu, T. H. Using ACME (Automatic Classification of Medical Entry) software to monitor and improve the quality of cause of death statistics. Fournal of Epidemiology and Community Health, 57 (2003-06-01 00:00:00 2003), 470-471.

[28] DIMDI - About Iris. DIMDI: German Institute of Medical Documentation and Information, City.

[29] Tao, C., Wongsuphasawat, K., Clark, K., Plaisant, C., Shneiderman, B. and Chute, C. G. Towards event sequence representation, reasoning and visualization for EHR data. In Proceedings of the Proceedings of the 2nd ACM SIGHIT International Health Informatics Symposium (Miami, Florida, USA, 2012). ACM, [insert City of Publication],[insert 2012 of Publication].

[30] Wang, T. D., Plaisant, C., Quinn, A. J., Stanchak, R., Murphy, S. and Shneiderman, B. Aligning temporal data by sentinel events: discovering patterns in electronic health records. ACM, City, 2008.

[31] Syed, H. and Das, A. K. Identifying Chemotherapy Regimens in Electronic Health Record Data Using Interval-Encoded Sequence Alignment. Springer, City, 2015.

[32] Casanova, I. J., Campos, M., Juarez, J. M., Fernandez-Fernandez-Arroyo, A. and Lorente, J. A. Using Multivariate Sequential Patterns to Improve Survival Prediction in Intensive Care Burn Unit. Springer International Publishing, City, 2015.

[33] Batal, I., Valizadegan, H., Cooper, G. F. and Hauskrecht, M. A pattern mining approach for classifying multivariate temporal data. IEEE, City, 2011

[34] Konias, S., Giaglis, G. D., Gogou, G., Bamidis, P. D. and Maglaveras, N. Uncertainty rule generation on a home care database of heart failure patients. City, 2003.

[35] Ordonez, C., Omiecinski, E., De Braal, L., Santana, C. A., Ezquerra, N., Taboada, J. A., Cooke, D., Krawczynska, E. and Garcia, E. V. Mining constrained association rules to predict heart disease. City, 2001.

[36] Liu, H. and Du, H. Stock Sequence Pattern Mining Method Based on SWI-GSP Algorithm. ACM, City, 2017.

[37] Zhang, D. and Zhou, L. Discovering golden nuggets: data mining in financial application. IEEE Transactions on Systems, Man, and Cybernetics, Part C (Applications and Reviews), 34, 4 (2004), 513-522.

[38] Vu, H. Q., Li, G., Law, R. and Zhang, Y. Travel Diaries Analysis by Sequential Rule Mining. Journal of Travel Research (2017), 0047287517692446.

[39] Tiwari, S. and Tiwari, L. K. Sequential Rule Mining in M-Learning Domain. International fournal of Computer Applications, 134, 3 (2016), 23-29.

[40] Pei, J., Han, J., Mortazavi-Asl, B., Wang, J., Pinto, H., Chen, Q., Dayal, U. and Hsu, M.-C. Mining sequential patterns by pattern-growth: The prefixspan approach. Knowledge and Data Engineering, IEEE Transactions on, 16, 11 (2004), 1424-1440.

[41] Lin, M.-Y. and Lee, S.-Y. Fast discovery of sequential patterns by memory indexing. Springer, City, 2002.

[42] Pradhan, G. N. and Prabhakaran, B. Association rule mining in multiple, multidimensional time series medical data. Fournal of Healthcare Informatics Research, 1, 1 (2017), 92-118.

[43] Vandromme, M., Jacques, J., Taillard, J., Hansske, A., Jourdan, L. and Dhaenens, C. Extraction and optimization of classification rules for temporal sequences: Application to hospital data. Knowledge-Based Systems, 122 (2017), 148-158.

[44] Concaro, S., Sacchi, L., Cerra, C., Fratino, P. and Bellazzi, R. Mining health care administrative data with temporal association rules on hybrid events. Methods of information in medicine, 50, 2 (2011), 166-179.

[45] Chaves, R., Górriz, J. M., Ramírez, J., Illán, I. A., Salas-Gonzalez, D. and GómezRío, M. Efficient mining of association rules for the early diagnosis of Alzheimer's disease. Physics in Medicine and Biology, 56, 18 (2011), 6047.

[46] Agrawal, R., Imieli, T., \#324, ski and Swami, A. Mining association rules between sets of items in large databases. In Proceedings of the Proceedings of the 1993 ACM SIGMOD international conference on Management of data (Washington, D.C., USA, 1993). ACM, [insert City of Publication],[insert 1993 of Publication].

[47] Srikant, R. and Agrawal, R. Mining sequential patterns: Generalizations and performance improvements. Springer, 1996.

[48] Wang, J., Han, J. and Li, C. Frequent closed sequence mining without candidate maintenance. Knowledge and Data Engineering, IEEE Transactions on, 19, 8 (2007), 1042-1056. 